\title{
EFECTO DE LA INTEGRACIÓN FÍSICA EN REGIONES PRÓXIMAS A LA INTEROCEANICA: UN ANÁLISIS ECONÓMICO PARA EL BRASIL Y PERÚ.
}

\author{
Jorge Luis Sanchez Arevalo* \\ Edgard Monforte Merlo ${ }^{\dagger}$
}

\begin{abstract}
Resumo
Este estudio tiene como objetivo probar empíricamente los objetivos de la integración física, específicamente los aspectos económicos. Ese tema pasa a ser relevante en las condiciones actuales, donde la búsqueda por nuevas formas de fortalecer las relaciones bilaterales y el comercio pueden servir de debate para el crecimiento de las regiones menos desarrolladas. Los resultados indican un beneficio no homogéneo de la integración física vinculado al mayor flujo comercial entre los dos países bajo estudio. El efecto frontera evidencia un efecto positivo para las regiones del Brasil y negativo para las regiones del Perú.
\end{abstract}

Palavras-chave: Integración física, Flujos comerciales, Modelo de gravedad.

\begin{abstract}
This study aims to prove empirically the objectives of physical integration, specifically economic aspects. This topic becomes relevant under current conditions, where the search for new ways to strengthen bilateral relations and trade can serve as a debate for the growth of less developed regions. The results indicate a non-homogenous benefit of the physical integration linked to the greater commercial flow between the two countries under study. The border effect shows a positive effect for the regions of Brazil and negative for the regions of Peru.
\end{abstract}

Keywords: Physical integration, Trade flows, Gravity model.

JEL classification: F13, F14, F15.

DOI: http://dx.doi .org/10.11606/1980-5330/ea137367

\footnotetext{
* Professor Adjunto - Universidade Federal de Mato Grosso do Sul. Doutor em Administração pela Universidade de São Paulo - Campus de Ribeirão Preto. E-mail: jsarevalo@usp.br

${ }^{\dagger}$ Professor Associado - Universidade de São Paulo. Departamento de Administração - Campus de Ribeirão Preto. E-mail: edgardmm@usp.br
} 


\section{Introducción}

La integración física entre los países visa fortalecer las relaciones bilaterales, lo que se refleja, entre otras consecuencias, en el aumento del intercambio comercial. Los supuestos vinculados a la relación de integración son los aspectos políticos, sociales y económicos. Los aspectos sociales son concebidos a partir de la necesidad percibida entre los países proponentes y en consecuencia, los aspectos políticos ganan relevancia porque son decisivos para la integración se lleva a cabo mediante común acuerdo. Ante tal, se destaca el tema de la integración y su efecto en las regiones fronterizas, que, dada la falta de este, genera desigualdad, comercio incipiente y por lo tanto tiene un coste social.

En América del Sur, este tema ganó notoriedad a principios de los años 90 con el desarrollo de la teoría del "nuevo regionalismo" impulsado por la Comisión Económica para América Latina - CEPAL (Cipoletta Tomassian 2009). El nuevo regionalismo, también llamado regionalismo abierto aparece como una nueva manera de incluir las nuevas teorías económicas del comercio internacional y el desarrollo en el contexto de la integración regional que se produjo en diversas regiones del mundo desde 1960. De forma específica, se entiende por nuevo regionalismo a la voluntad conjunta de los países de América Latina en superar los efectos ocasionados por la larga crisis de los años 1980, por medio de la apertura comercial y de la implantación de políticas para la atracción de inversión, y así proporcionar a la región una mejor inserción del mercado internacional (Oliveira 2014, Reis et al. 2014, Ethier 1998).

Importante mencionar que las ideas clásicas de Smith y Ricardo son fundamentales para llegar a las instancias en la definición y diseño de la teoría del "nuevo regionalismo", y esa última teoría fortalece el proceso de integración entre los países latinoamericanos. Además, la teoría de la competitividad de las naciones de Porter, también fue crucial en ese proceso, ya que por medio de esa teoría estudios sobre el comercio internacional en el área de administración fueron llevados a cabo.

En vista de la necesidad de integración entre los países de América del Sur, se creó la Iniciativa para la Integración de la Infraestructura Regional de América del Sur, o simplemente - IIRSA, en agosto del 2000. La creación de ese programa es el resultado del reconocimiento por el cual los países de la región señalan que el esfuerzo de integración a través de la reducción o el final de las barreras aduaneras regionales entre países no es suficiente (Sierralta 2014). En vista de esto, teniendo en cuenta la realidad de la infraestructura de los países de América del Sur, es necesario planificar la construcción de medios físicos (infraestructura) que ayuden a una mejor circulación de bienes, servicios y personas entre los países vecinos.

Es en ese contexto que se centra este artículo, que estudia la integración física entre Brasil y Perú, desde un punto de vista económico. De esta manera, se toma como objeto de estudio la carretera interoceánica que une los dos países. En vista de eso, centrándose en la experiencia brasileña y peruana, se evalúa los principales objetivos y resultados para los cuales se ha propuesto este proceso de integración.

El propósito de este estudio es avanzar en la investigación sobre la influencia de la integración física en las relaciones económicas entre países vecinos. Estudios de esa naturaleza para los dos países (Brasil y Perú) son escasos en la literatura, así se enfatiza la importancia de este. Por lo tanto, este estudio tiene como objetivo contribuir a una mayor comprensión de la realidad de esta 
integración, usando técnicas y métodos de análisis que tienen su sustento en la teoría económica.

\section{Enfoque y Debates en los Estados Fronterizos Después de la Conclusión de la Interoceânica 2011-2015}

\subsection{Contexto peruano}

En 2011, después de la finalización de las obras, la discusión sobre el uso de la carretera por parte de los exportadores e importadores, se centraba en el requisito de contar con unidades de transporte, ya sean propias o de terceros, teniendo en cuenta que esta sería una de las características en la cual los costes serían afectados. En una información publicada por el Ministerio de Transporte y Comunicaciones de Perú - MTC (2011), se reportó que el tránsito de las unidades de transporte se incrementó de 60 a 200, en la ruta Urcos - Inhambari (estado de Cusco/estado de Madre de Dios), trayecto por donde pasa la carretera, medida por el número de rutas comerciales en 2011. Así pues, se verifica que la mejora de la infraestructura y nuevas rutas han fomentado la conexión entre ciudades.

En consecuencia, se espera que el comercio se impulse en las regiones y/o ciudades donde pasa la carretera. Además, sirva de condición para que productos de la Amazonía llegan más rápido a las ciudades situadas en la costa. En general, se espera que los productos nacionales puedan ser comercializados a un costo menor, menos tiempo y exhiban ganancias de competitividad. Según Felipa (2012), la visión de los empresarios peruanos era que en el mediano plazo haya un aumento en las ventas de productos agrícolas tales como aceitunas, cebollas y patatas, que son productos de alta demanda en Acre y Rondonia. Lo mismo puede ocurrir con bienes semi-manufacturados, tales como productos para la industria de la construcción, de lo cual el Perú exporta a otros estados como Mato Grosso, Goiás, Sao Paulo y Paraná.

Con base en la Tabla 2, a continuación, se observa que el número de habitantes en Madre de Dios, se ha incrementado a tasas más altas en comparación con otros estados que forman parte de la microrregión sur de Perú, con un valor del 36\% desde 2000 hasta 2010 y de $11 \%$ de 2010 a 2014. En el mismo departamento de Madre de Dios, de 2010 a 2014, el número de nuevas empresas casi se duplicó al pasar de 6,592 en 2010 a 11,400 en 2014. En la cuestión salarial, ese departamento también muestra una mejor remuneración en comparación con los demás, se observó que el salario medio de personas empleadas fue de US\$ 529.2 en 2010 lo cual aumentó a US\$ 650.9 en 2014. Ese valor de ingreso es similar a lo observado en el departamento de Moquegua, siendo que en el contexto peruano ese departamento es sinónimo de poder mineral y alta concentración del ingreso. En concreto, el Perú es considerado uno de los mayores productores de oro, plata y cobre en el mundo, las reservas se concentran principalmente en las regiones costeras y el sur de ese país.

Por lo tanto, se observa que la carretera, así como otros factores tales como el aumento del número de empresas, también ha dado lugar a una mejora en los ingresos en los departamentos próximos. En resumen, la construcción y pavimentación de carreteras ha hecho más que cualquier otra medida, reducir la pobreza en el Perú en los últimos años.

De acuerdo con el contexto presentado y reflexionando sobre ella, sigue la primera hipótesis de investigación: El flujo comercial entre Brasil y Perú tiene 
Cuadro 1: Departamentos del Perú localizados cerca de la carretera: principales productos de exportación

\begin{tabular}{|c|c|}
\hline Departamentos & Principales productos de exportación \\
\hline Apurímac & a) Agricultura: papas, cebollas, maíz y cebada. \\
\hline Arequipa & $\begin{array}{l}\text { a) Minerales: cobre, el oro y la plata; b) Pesca y hari- } \\
\text { na de pescado; c) Agricultura: alfalfa, patatas, arroz, } \\
\text { cebolla y ajos; d) Carne de ganado y lácteos. }\end{array}$ \\
\hline Cusco & a) Turismo; b) Granos de cereales; c) Bovinos. \\
\hline Ica & $\begin{array}{l}\text { a) Minerales: cobre, hierro y estaño; b) Agricultura: } \\
\text { uvas, espárragos y paltas; c) Querosene y gas. }\end{array}$ \\
\hline Madrede Dios & a) Mineral: oro; b) Caucho, madera y castaña. \\
\hline Moquegua & $\begin{array}{l}\text { a) Minerales: cobre y plata; b) Pesca y harina de pes- } \\
\text { cado; c) Agricultura: alfalfa, papa, maíz y aceitunas; } \\
\text { d) Camélidos. }\end{array}$ \\
\hline Puno & $\begin{array}{l}\text { a) Minerales: estaño, oro y plata; b) Agricultura: pa- } \\
\text { pas, alfalfa, avena; c) Plátano y naranja; d) Bovinos } \\
\text { y lácteos, camélidos; e) Industria textil. }\end{array}$ \\
\hline Tacna & $\begin{array}{l}\text { a) Minerales: cobre y plata; b) Pescado en conserva y } \\
\text { frutos del mar; c) Agricultura: alfalfa, cebolla, papas } \\
\text { y aceitunas; d) Avícola, porcinos y camélidos. }\end{array}$ \\
\hline
\end{tabular}

Fuente: SUNAT (2016).

Cuadro 2: Departamentos del Perú localizados cerca de la carretera: Población, Ingresos y Empresas

\begin{tabular}{l|r|r|r|r|r|r}
\hline \multirow{2}{*}{ Departamentos } & \multicolumn{4}{|c|}{2010} & \multicolumn{3}{c}{2014} \\
\cline { 2 - 7 } & $\begin{array}{c}\text { Var. } \\
\text { Pob.1 }\end{array}$ & Ingreso & Empresas & $\begin{array}{c}\text { Var. } \\
\text { Pob.2 }\end{array}$ & Ingreso & Empresas \\
\hline Apurimac & $1 \%$ & 224.4 & 7,955 & $8 \%$ & 296.4 & 13,325 \\
\hline Arequipa & $12 \%$ & 373.5 & 69,297 & $5 \%$ & 503.3 & 91,181 \\
\hline Cusco & $9 \%$ & 274.5 & 41,688 & $3 \%$ & 380.8 & 62,537 \\
\hline Ica & $14 \%$ & 327.4 & 29,337 & $4 \%$ & 418.1 & 41,984 \\
\hline Madre de Dios & $36 \%$ & 529.2 & 6,592 & $11 \%$ & 650.9 & 11,400 \\
\hline Moquegua & $13 \%$ & 537.4 & 8,858 & $4 \%$ & 642.3 & 10,790 \\
\hline Puno & $11 \%$ & 208.2 & 22,653 & $4 \%$ & 309.9 & 33,286 \\
\hline Tacna & $19 \%$ & 398.2 & 20,549 & $5 \%$ & 546.2 & 26,457 \\
\hline
\end{tabular}

Fuente: Preparado a partir de INEI (2016)

Var. Pop. 1 = variación porcentual de la tasa poblacional entre 2000-2010,

Var. Pop. 2 = variación porcentual de la tasa poblacional entre 2010-2014,

Ingreso = renta mensual promedio de personas ocupadas en 2010 y 2014. Valores

corrientes en dólar calculado usando el tipo de cambio anual disponible por el USDA (2.83

en 2010 y 2.84 en 2014).

Empresas = número de empresas formales en 2010 y 2014. 
una tasa promedio mayor de crecimiento de 2011 a 2015 en comparación con 2000-2010. A saber, los estados vecinos/fronterizos muestran ganancias de comercio después de la terminación de la carretera, para lo cual, el transporte por carretera y las distancias son cruciales para explicar ese problema.

Como consecuencia de la hipótesis descrita y con base en el modelo de gravedad utilizado en este estudio, se determina que el comercio entre los estados de ambos países es directamente proporcional al PIB e inversamente proporcional a la distancia entre ellos. Se tomó como factor la "distancia" entre las ciudades capitales como determinante en el comercio. Junto a esto, existe la variable "frontera" en el que se determina el beneficio de la integración para los estados ubicados en el eje de la carretera.

En la visión de este estudio, se argumenta que el comercio con el mercado brasileño facilitado por las condiciones de infraestructura genera al Perú más propensión a exportar sus productos por carretera, probablemente con un flete más bajo y pudiendo competir con los precios en el mercado brasileño ${ }^{1}$.

\subsection{Contexto brasileño}

En 2011 ya con los trabajos terminados y con la carretera en uso, en el escenario brasileño (en los estados ${ }^{2}$ próximos a la carretera, principalmente), el resultado esperado de ese proyecto aún estaba asociado al de servir como medio para una mayor aproximación cultural, el turismo y el comercio, lo que traería beneficios para ambos países. Además, de servir como atajo para el flujo de las exportaciones a China, esa visión ya era debatida mucho antes, desde el inicio de la construcción de ese proyecto.

No obstante, se observó que el proceso de integración ha comenzado a dar sus primeros frutos, este hecho se verifica en la observación del cambio relacionado con el tiempo de viaje para el transporte entre las ciudades cerca de la carretera. El mismo que se esperaba que se vea facilitada por la nueva carretera (MTUR, 2011).

También, se verificó una relación más estrecha entre los empresarios situados en Acre y Rondonia con los peruanos, siendo el ambiente notoriamente optimista, lo cual visa aumentar el flujo de comercio de las regiones. Ese contexto es resultado de la promoción del evento "Expo Perú Amazonas" en 2012, que es fruto de una asociación entre la Comisión de Promoción de Exportación y el Turismo del Perú (PROMPERÚ) y la Federación de Industrias del Estado de Rondonia (FIERO).

Como consecuencia, en la escena brasileña comenzó a ser discutido con más detalle los beneficios que tanto Brasil y los estados cerca de la frontera pueden obtener en el comercio con Perú. Así, productos de mayor demanda en el Perú y/u oferta en Brasil, comenzaron a publicarse en los medios de comunicación, entre los que están: a) el pescado y harina de pescado, b) los minerales como el fosfato, c) los productos textiles, d) petróleo y gas natural, y) de cemento f) las uvas; productos que los estados de Acre, Rondonia y Mato Grosso compran del Perú. A cambio, los mismos estados venden fosfato de calcio, aceitunas en conserva, película de polipropileno y prendas de vestir,

\footnotetext{
${ }^{1}$ Carrere (2006) y Porto et al. (2015), argumentan que en general, las medidas de facilitación del comercio en su conjunto ayudarán a los países a mejorar su desempeño comercial.

${ }^{2}$ En este estudio se considera la división administrativa del Perú en departamentos y el de Brasil en estados.
} 
Cuadro 3: Estados del Brasil localizados cerca de la carretera: principales productos de exportación

\begin{tabular}{c|c}
\hline Estados & \multicolumn{1}{c}{ Principales productos de exportación } \\
\hline Acre & $\begin{array}{l}\text { a) Agricultura: arroz, maíz, yuca, plátano y frijol; b) } \\
\text { Bovinos y lácteos; c) Caucho, castañas, madera; d) } \\
\text { Porcinos y aves. }\end{array}$ \\
\hline Mato Grosso & $\begin{array}{l}\text { a) Agricultura: soja y maíz; b) El ganado vacuno y } \\
\text { pollo; c) Mineral: hierro; d) Celulosa, alcohol, azú- } \\
\text { car, aceites vegetales. }\end{array}$ \\
\hline Rondônia & $\begin{array}{l}\text { a) Agricultura: soya, maíz, arroz y yuca; b) Bovinos y } \\
\text { lácteos; c) Mineral: tungsteno y oro; d) Castaña, ma- } \\
\text { dera y carbón vegetal; e) Aves, frutas y horticultura. }\end{array}$ \\
\hline
\end{tabular}

Fuente: SUNAT (2016).

principalmente (MDIC 2016). Por lo que se entiende, que el intercambio comercial entre los estados localizados cerca de la carretera se va fortaleciendo cada vez más, algo que refuerza la primera hipótesis.

Asimismo, se observan cambios significativos en el escenario económico y social de los estados localizados cerca de la carretera, más específicamente en Acre, como se ve en la Tabla 4. En Acre entre 2000 y 2010 la tasa de crecimiento poblacional fue $31 \%$ mayor en comparación con los otros dos estados, sin embargo, en lo que se refiere a creación de nuevas empresas se nota un descenso.

En el caso de Rondônia, se observa un mayor crecimiento de la tasa poblacional entre 2010 y 2014 con un $12 \%$, a expensas de los otros dos estados, sin embargo se nota una disminución en la creación de nuevas empresas. En Mato Grosso, el crecimiento de la tasa poblacional sigue un ritmo menor en comparación con los otros dos estados entre 2010 y 2014 y en cambio se nota un crecimiento en relación con la creación de nuevas empresas. Por el lado de los ingresos, hay un aumento en los tres estados de 2010 para 2014, lo cual se explica por aumentos del salario mínimo, a pesar de que la moneda nacional se tenga devaluado (por razones de comparación se utiliza el valor en US\$ para el salario mínimo).

La idea inicial con el proyecto de la interoceánica, como se señaló anteriormente, era la de ser un corredor para las exportaciones, principalmente de cereales, de Brasil al mercado asiático. En ese tramo gano fuerza el debate en lo que se refiere a de servir como impulsor para fortalecer el flujo del comercio. Situaciones habituales de debate eran casos como el reclamo de autoridades, tanto brasileñas como peruanas, acerca de la burocracia (retraso en el servicio y un menor número de servidores), siendo que en el lado brasileño el problema se considera mayor. Por otra parte, el alto precio que se cobra por las compañías brasileñas de transporte hace que algunas compañías brasileras de comercio alquilen transportadores en el propio Perú, que cobran mucho menos por el servicio.

Considerando lo antes expuesto, sigue la siguiente hipótesis de investigación de dos: La integración física entre los dos países es más beneficioso para Perú con respecto al comercio, teniendo en cuenta la importancia del mercado brasileño para los productos exportados desde el Perú. A saber, la exportación 
Cuadro 4: Estados del Brasil localizados cerca de la carretera: Población, Ingresos y Empresas

\begin{tabular}{|c|c|c|c|c|c|c|}
\hline \multirow[b]{2}{*}{ Estados } & \multicolumn{3}{|c|}{2010} & \multicolumn{3}{|c|}{2014} \\
\hline & $\begin{array}{c}\text { Var. } \\
\text { Pob. } 1 \\
\end{array}$ & Ingreso & Empresas & $\begin{array}{c}\text { Var. } \\
\text { Pob. } 2 \\
\end{array}$ & Ingreso & Empresas \\
\hline Acre & $31 \%$ & 898.3 & 10,082 & $8 \%$ & 928.5 & 9,297 \\
\hline Rondonia & $13 \%$ & 869.3 & 34,374 & $12 \%$ & 886.7 & 34,307 \\
\hline Mato Grosso & $21 \%$ & 811.4 & 86,476 & $6 \%$ & 894.9 & 92,489 \\
\hline \multicolumn{7}{|c|}{$\begin{array}{l}\text { Fuente: Preparado a partir de IBGE (2016). } \\
\text { Var. Pop. } 1 \text { = variación porcentual de la tasa poblacional entre } 2000-2010, \\
\text { Var. Pop. } 2 \text { = variación porcentual de la tasa poblacional entre } 2010-2014 \text {, } \\
\text { Ingreso = renta mensual promedio de personas ocupadas en } 2010 \text { y } 2014 \text {. Valores corrientes } \\
\text { en dólar calculado usando el tipo de cambio anual disponible por el USDA ( } 1.76 \text { en } 2010 \text { y } \\
2.35 \text { en } 2014) \text {. } \\
\text { Empresas = número de empresas formales en } 2010 \text { y } 2014\end{array}$} \\
\hline
\end{tabular}

de productos del Perú a Brasil tiene mayor ganancia de competitividad ${ }^{3}$.

En adición a lo ya mencionado, si bien la comunidad local ha seguido el desarrollo de la carretera, la cual visa fomentar el comercio, y teniendo en cuenta las diferencias que existen en el entorno empresarial brasileño, se espera que tal proyecto sea más beneficioso para el Perú; y específicamente a los estados situados cerca de la carretera para los países.

\section{Metodología}

\subsection{Modelo de gravedad a ser estimado}

El enfoque econométrico para análisis de estudios que implican la estimación de modelos gravitacionales puede tomar muchas formas. Ante lo cual, se tiene el método de los Mínimos Cuadrados Ordinarios - "pooled" (datos combinados), el método de efectos fijos y aleatorios, que se calcula a partir de la adopción de un modelo de datos de panel, y el método "Poisson" o "PseudoMaximum-Likelihood" - PPML 4 . Por lo general, esos tres enfoques son los más comunes en este tipo de análisis.

El método de datos combinados (MCO) es el más simple y frágil (frente a la crítica), ya que en ese no se considera los efectos individuales (políticas económicas adoptadas) de los países, lo que resulta en problemas de sesgo en los datos colectados. También, la estimación de este método no es tan precisa en comparación con los otros mencionados.

Un segundo enfoque sería el uso de la metodología en datos de panel. En este caso, también los modelos estimados son propensos a las críticas por no tomar en cuenta los efectos fijos y aleatorios a través de una serie de tiempo histórico. La estimación en este caso muestra las características individuales de cada país (método de efectos fijos). Esa metodología es ampliamente utilizada en los estudios relacionados al comercio internacional, ya que permite

\footnotetext{
${ }^{3}$ Argumento provado pelo autor em estudo anterior, Arevalo \& Merlo (2017)

${ }^{4}$ Detalles ver Weeks (2014), Mier (2008) y Egger (2005), el último autor además de destacar la importancia de los efectos fijos, también destaca la estimación por medio del modelo HausmanTaylor, la cual según el autor merece atención en la estimación de los modelos de gravedad
} 
controlar la heterogeneidad entre países, como resultado de las diferencias, considerando casos no observables debido a naturalezas diversas ${ }^{5}$.

Sin embargo, Silva \& Tenreyro (2006) propusieran el uso del "Pseudo-MaximumLikelihood" - PPML, con el fin de hacer frente a los problemas de falta de observaciones en la muestra y los valores nulos, lo que representa una mejora sobre el método de efectos fijos. PPML, por lo tanto, se utilizará este estudio, ya que puede ser considerado superior a los efectos fijos, para la introducción de estimaciones no sesgadas en sus simulaciones de Monte Carlo. Ante tal, en este estudio fue utilizado el PPML ya que puede ser considerado superior a los efectos fijos, porque presenta estimativas no sesgadas en simulaciones de Monte Carlo.

En retrospectiva, el uso del método de la gravedad se puede decir que el modelo fue utilizado por primera vez por Tinbergen \& Hekscher (1962) y más tarde por Linnemann (1966), que tiene su origen racional en la teoría de la gravedad de la física. En general, el modelo de gravedad indica que el flujo comercial está determinado por fuerzas de atracción (tamaño de la economía en el modelo económico y la masa corporal en la teoría de la física) y las fuerzas de repulsión (en la economía, distancias entre los países y en física distancia entre los cuerpos). A fin de proporcionar una base teórica para este tipo de análisis, Anderson (1979) desarrolló un contexto teórico basado en las preferencias Cobb-Douglas y la elasticidad de sustitución constante (Función CES), siendo ese enfoque, en ambos casos, la diferenciación de productos por país de origen.

Anderson \& Van Wincoop (2003) actualizan el método de gravedad, de lo cual se puede considerar como un enfoque estándar para el comercio internacional al utilizar esta herramienta de análisis. Los autores, con base en la teoría existente, desarrollaron un modelo derivado de las preferencias de tipo CES (elasticidad de sustitución constante) sujetas a limitación presupuestaria y adecuando a las condiciones de equilibrio de mercado. Así pues, según los autores, los flujos comerciales no sólo dependen de los costes entre los países socios, sino también del costo de comercio en otros países asociados. En el caso de este estudio, se consideró el flujo de comercio entre los dos países a la luz de la integración física y los factores externos que afectan el comercio.

Por lo tanto, como se ha mencionado anteriormente, se analiza el comercio entre ambos países por origen y destino. Es decir, el flujo de comercio entre los estados brasileños que exportan al Perú y viceversa. En vista de esto, el objetivo es comprobar el flujo del comercio vía transporte de carretera, visando a que las regiones fronterizas por medio de la variable "front" tengan coeficientes con signos positivos y significativos. En esa misma línea, se visa analizar el "efecto distancia", lo que indica que cuanto más cerca están los estados de la carretera, mayor será el comercio, de lo contrario, el mismo tiende a disminuir. Con respecto a la variable PBI de ambos países, se espera que la incidencia de esa variable sea positiva en relación con el comercio.

Para la estimación de las siguientes ecuaciones mencionadas a seguir fue utilizado un tratamiento por varios métodos, con el objetivo de superar susceptibles críticas al método Pooled. Para varios métodos, además del Pooled, se tiene el método de efectos fijos, efectos aleatorios y PPML. En concreto, los mé-

\footnotetext{
${ }^{5}$ Mencionam-se os estudos de Kapuya et al. (2014); Otor (2017), Cheng et al. (2005) y Magee (2008)
} 
todos fijos y aleatorios y PPML tienen resultados más precisos, los cuales son corroborados por las pruebas estadísticas que se muestran en los resultados.

Ecuaciones estimadas por varios métodos:

Brasil:

$$
Y_{i j t}=L g d p 1_{i t}+L d 1_{i j}+L t c 1_{i t}+F r o n t 1_{i j}+\mu_{i j t} 1
$$

Perú:

$$
Y_{i j t}=L g d p 2_{i t}+L d 2_{i j}+L t c 2_{i t}+\text { Front } 2_{i j}+\mu_{i j t} 2
$$

Donde:

Yijt = Corresponde al flujo de comercio entre los estados del Brasil y del Perú y viceversa.

Lgdp1it = corresponde al PBI de los estados de origen, país de exportación, Brasil en este caso;

Lgdp2it = Corresponde al PBI de los estados de origen, país de exportación, Perú en este caso;

Ld1ij = Corresponde a las distancias entre los estados del Brasil al Perú. Se consideran las distancias bilaterales hasta la mayor concentración de los ingresos del país. Esa metodología fue establecida por el CEPII, la cual es ampliamente utilizada en investigaciones de esta naturaleza;

Ld2ij = Se corresponde con la distancia entre los estados de Perú a Brasil.

Ltc1it = Tipo de cambio real brasileño/nuevo sol;

Ltc2it = Tipo de cambio nuevo sol/real brasileño;

Front $1 \mathrm{ij}=$ Se considera la dummy $=1$ para los estados del Brasil por donde pasa la carretera, de lo contrario $=0$;

Front $2 \mathrm{ij}=$ Se considera la dummy $=1$ para os Departamentos del Perú por donde pasa la carretera, de lo contrario $=0$.

En general, las dos ecuaciones tienen variables comunes, que sólo se diferencian por el número (1) para Brasil y el numeral (2) para el Perú.

\subsection{Fuente de los datos}

Los datos de exportación para la estimación del modelo de gravedad se obtuvieron de las siguientes instituciones: del Brasil se obtuvieron de la página Alice Web, disponible por el MDIC (2016). Los datos de exportación de Perú se obtuvo de la página del SIICEX (2016) disponible por el de Comercio Exterior y Turismo (2016) y por la SUNAT (2016).

El Producto Bruto Interno (PBI) fue utilizado como proxy del ingreso. Los valores corrientes del PBI se obtuvieron de la información divulgada por el IBGE (2016) del Brasil y el INEI (2016) del Perú.

La metodología de la distancia geográfica entre los países y sus regiones/estados sigue el modelo establecido por el Centre d'Études Prospectives et d'Informations Internationales (2016). Ese instituto en sus base de datos "distancias" presenta la información de varias variables geográficas que por lo general se consideran en los modelos de gravedad, entre ellos se incluye la "distancia bilateral" entre los países. En el cálculo de la "distancia" se consideran las latitudes y longitudes de las ciudades más importantes. En el caso de Brasil, San Pablo y Río de Janeiro, y en el caso del Perú, Lima. 
Los datos del tipo de cambio se obtuvieron de la información publicada por United Nations (2016).

\section{Resiltados}

\subsection{Resultados referente a los modelos estimados}

Con respecto al método pooled es importante tener en cuenta algunas críticas en algunos casos este método puede tener estimaciones sesgadas, que se explica por el hecho de no tener en cuenta los efectos específicos de cada estado de los países de análisis. Para comparar los resultados del Pooled con el método de efectos aleatorios, se utiliza la prueba del multiplicador de Lagrange (LM) propuesto por Breusch \& Pagan (1980). En las Tablas 5 y 6 se observa que por medio de la prueba LM la hipótesis nula de que no hay efectos aleatorios es rechazada, que sirve como prueba de que el método pooled no es idóneo. En la visión de Cheng et al. (2005) y Weeks (2014) los resultados estimados por el modelo pooled deben ser desconsiderados.

Con respecto al "efecto fijo", al comparar los resultados de ese modelo con el del "efecto aleatorio" se usa la prueba de Hausman. La hipótesis nula es que la diferencia de los coeficientes estimados no es sistemática, es decir, exogeneidad entre $\alpha_{i} y x_{i}$ (la cuestión es si los efectos individuales $\alpha_{i}$ están o no correlacionados con los regresores $x_{i}$ ). Como se rechaza esa hipótesis (véase las Tablas 5 y 6), se verifica que el método de los efectos fijos es más adecuado. Intuitivamente, se puede decir que este resultado tiene sentido, cada vez que se considera que hay efectos individuales no observables en cada uno de los estados, lo cuales son determinantes para el flujo comercial. Es decir, cada estado y/o departamento en particular, de cada país en análisis tienen un comportamiento propio y diferente de los demás.

Por último, con el fin de determinar si el PPML es preferible en comparación a los otros métodos estimados (Tablas 5 y 6), se utiliza la prueba RESET formulado por Ramsey (1969). La hipótesis nula es que el modelo no muestra variables omitidas. En vista de esto, a partir de los métodos estimados, el PPML es el único al que no se puede rechazar esa hipótesis, de esa manera, es posible concluir que es el método más apropiado para la estimación del modelo de gravedad, tal como sustentado por Silva \& Tenreyro (2006); Silva \& Tenreyro (2010); Silva \& Tenreyro (2011) en sus análisis acerca del comercio internacional.

La utilización del modelo en datos de panel parte de la premisa de que existían puntos coherentes en la literatura que permitieron seleccionar un método de estudio riguroso que relacionara los fenómenos económicos en el ámbito del intercambio comercial entre países, siendo el modelo gravitacional adecuado para tal finalidad.

\subsection{Resultados encontrados}

En la Tabla 5 se observa los resultados de la ecuación (1), donde se considera todos los estados de Brasil que exportan al Perú a través de diferentes medios de transporte. Se observa que, en todos los métodos, el coeficiente de la variable "Lgdp1" muestra estadísticas significativas y signos esperados. Sin embargo, el coeficiente de las variables "Ld1" y "Ltc1" a pesar de que el signo es esperado, las mismas no muestran significación estadística. 
El coeficiente de la variable "Lgdp1" indica que la oferta de exportación de Brasil a Perú se comporta positivamente en relación con el aumento de los ingresos de Brasil. Se esperaba ese resultado toda vez que se justifica teóricamente, ya que el aumento de las exportaciones causa aumento en el ingreso del país exportador. En el caso específico del método de PPML, por ejemplo, un aumento del $10 \%$ en los ingresos, aumenta el comercio en $2.42 \%$ aproximadamente.

El coeficiente negativo de la variable "Ld1" era esperado, lo que indica que cuanto mayor son las distancias, el intercambio comercial tiende a disminuir. Ese resultado se corrobora con estudios anteriores tales como Anderson \& Van Wincoop (2004), Brun et al. (2005), Blum \& Goldfarb (2006).

El coeficiente negativo de la variable "Ltc1" indica que dado el aumento de la tasa de cambio (real/nuevo sol), el efecto es una disminución de la oferta de exportación de Brasil a Perú, dado que mayor dinero en moneda extranjera (Nuevo Sol) sería recibido. Ese comportamiento denota no ser atractivo al Brasil, de esa forma se verifica que la moneda de Perú no es atractiva en el mercado brasileño. En vista de esto, se valida la segunda hipótesis, que el mercado brasileño es atractivo para Perú, y lo contrario no es cierto. Históricamente el real brasileño se mantuvo apreciado frente al nuevo sol, y este comportamiento ha cambiado ligeramente en 2015 específicamente el nuevo sol se ha apreciado frente al real.

El resultado esperado del coeficiente de la variable "Front1" indica que el comercio es crucial para los estados ubicados en la zona fronteriza (por donde pasa la carretera Interoceánica). Este resultado explica el relativo crecimiento del comercio que los estados de Acre, Rondonia y Mato Grosso tienen con Perú, la misma que poder ser comparado (en relación) con el promedio de exportación de todos los estados que mantienen relaciones comerciales con Perú.

Aún en la Tabla 5, llaman la atención la magnitud de los coeficientes de cada variable en el método de PPML en comparación con las otras estimaciones. Se observa que la elasticidad de cada una de las variables, incluyendo la variable Dummy (Front1), del método PPML está por debajo de la media de los coeficientes de las otras estimaciones. En general, por el método PPML se verifica hay un efecto menor con relación al propuesto por el promedio de los otros métodos estimados.

Teniendo en cuenta la estimación por el método PPML, los resultados de los coeficientes para las variables "Lgdp1", "Ld1", "Ltc1" y "Front1" eran esperados, por lo tanto, apoya la evidencia empírica descritas en estudios anteriores, que se justifican por la naturaleza teórica de la determinación de los flujos comerciales.

Por el principio de la parsimonia, se considera que la estimación de la ecuación por diversos métodos y específicamente el método PPML es el más adecuado para este tipo de análisis considerando que ese método permite la eliminación de posible existencia de heterocedasticidad en el modelo, además, la adecuación del método de PPML se ve reforzada por el valor de la prueba Reset.

Considerando os resultados de la ecuación (2), en la que tiene en cuenta todos los estados del Perú que exportan al Brasil a través de diferentes medios de transporte. Se observa una similitud con respecto al valor significativo de los coeficientes y divergencia en la elasticidad de cada uno de los coeficientes estimados en todos los métodos. Por lo tanto, en este punto de la discusión se 
Cuadro 5: Resultados de la estimación de la ecuación (1) por varios métodos caso general para todos los estados de Brasil que exportan al Perú

\begin{tabular}{|c|c|c|c|c|}
\hline Variables & Pooled & Efecto Fijo & Efecto Aleatório & PPML \\
\hline LGDP1 & $\begin{array}{l}1.908^{* * *} \\
(0.081)\end{array}$ & $\begin{array}{l}1.168^{* * *} \\
(0.119)\end{array}$ & $\begin{array}{l}1.359^{* * *} \\
(0.109)\end{array}$ & $\begin{array}{l}0.242^{\text {*** }} \\
(0.018)\end{array}$ \\
\hline Ld1 & $\begin{array}{c}-0.132 \\
(0.466)\end{array}$ & - & $\begin{array}{l}-0.866^{*} \\
(1.193)\end{array}$ & $\begin{array}{c}-0.148^{*} \\
(0.103)\end{array}$ \\
\hline Ltc1 & $\begin{array}{c}-0.727 \\
(0.574)\end{array}$ & $\begin{array}{c}-0.053 \\
(0.821)\end{array}$ & $\begin{array}{c}-0.219^{*} \\
(0.471)\end{array}$ & $\begin{array}{c}-0.197^{*} \\
(0.125)\end{array}$ \\
\hline Front1 & $\begin{array}{r}0.748^{*} \\
(0.425) \\
\end{array}$ & - & $\begin{array}{c}-0.565 \\
(0.565) \\
\end{array}$ & $\begin{array}{c}0.191^{* *} \\
(0.191) \\
\end{array}$ \\
\hline Constante & $\begin{array}{c}-24.828^{* * *} \\
(4.634)\end{array}$ & $\begin{array}{c}-14.911^{*} \\
(11.281) \\
\end{array}$ & $\begin{array}{l}-8.269 \\
(10.729) \\
\end{array}$ & $\begin{array}{l}-3.468^{* * *} \\
(1.075)\end{array}$ \\
\hline Observações & 320 & 320 & 320 & 320 \\
\hline Grupos (Estados) & 20 & 20 & 20 & 20 \\
\hline$R^{2}$ & 0.677 & 0.347 & 0.356 & 0.138 \\
\hline Teste F & $165.38^{* * *}$ & & & \\
\hline Prueba Chow & & $12.92^{* * *}$ & & \\
\hline Teste de Hausman & & $16.04^{* * *}$ & & \\
\hline Prueba LM & & & $267.78^{* * *}$ & \\
\hline Log Likelihood & -619.978 & -592.231 & & -714.191 \\
\hline Teste Reset & $53.75^{* * *}$ & $20.73^{* * *}$ & $19.92^{* * *}$ & 0.94 \\
\hline
\end{tabular}

Fuente: Elaboración propia.

Nota: Los errores estándar aparecen entre paréntesis. Para los métodos de EF y EA, se informa de la R-cuadrado "within groups"

${ }^{* * *}$ Significancia a $1 \% ;{ }^{* *}$ Significancia a $5 \%$; Significancia a $10 \%$.

foca solamente en los resultados obtenidos por el método PPML, que a su vez es visto como el método más preciso para explicar el problema en cuestión, la cual se apoya en el valor de prueba Reset.

Mediante el método PPML se observa que todos los coeficientes de las variables del modelo tienen resultados esperados y la significación estadística, a excepción de la variable "Front2", que aunque mostrando la significación estadística en el signo del coeficiente es negativo y por lo tanto no esperado. Por lo tanto, se determina que la integración física plasmada en una mejorada de la infraestructura para el transporte por carretera no ha traído el resultado esperado para las regiones situadas en las zonas fronterizas de Perú. Este resultado se explica por el bajo rendimiento de las exportaciones que los departamentos próximos a la frontera con el Brasil, siendo que esas regiones no siguen (en promedio) el crecimiento real del comercio de los otros departamentos del Perú. Aun así, aunque las exportaciones de Madre de Dios y Puno no sean significativas, existe un aumento desde 2011, algo que no sucedió años anteriores.

Teniendo en cuenta el resultado de "Lgdp2" se comprueba que el aumento de la oferta exportable del Perú a Brasil se explica positivamente por el aumento de los ingresos del país exportador. En concreto, usando el método de PPML, se verifica que un aumento del $10 \%$ en los ingresos, tiende a aumentar el comercio en $0.92 \%$ aproximadamente.

El resultado positivo del coeficiente de la variable " $\mathrm{Ld} 2$ " indica que las 
distancias de los estados de Perú a Brasil no son condiciones para que el intercambio comercial se lleve a cabo. Este resultado contradice la literatura, cada vez que se espera que el comercio se fortalece cuando hay una proximidad o cuando se favorecen las condiciones del comercio, tales como: infraestructura adecuada para el transporte, el coste y el tiempo de transporte, entre otros. En este caso, se verifica que los departamentos con los resultados de exportación más altos del Perú se encuentran en la región costera y por lo tanto más lejos en comparación con otros departamentos que exportan a Brasil, resaltando que los departamentos situados en la región costera de Perú son el más desarrollado en ese país. Por lo tanto, este argumento justifica el valor positivo del coeficiente de la variable " $\mathrm{Ld} 2$ "6

El resultado positivo del coeficiente de la variable "Ltc2" indica que dado el aumento de la tasa de cambio (Nuevo Sol/Real) el efecto es un aumento de la oferta de exportación de Perú a Brasil. Este comportamiento muestra que es preferible al Perú mantener su moneda depreciada frente al real brasileño. Históricamente el real brasileño se mantuvo apreciado frente al nuevo sol, sólo en el 2015 ese comportamiento ha cambiado, el tipo de cambio comenzó a fluctuar en un promedio de 0.95 centavos, es decir 1 real brasileño equivalente a 0.95 centavos de nuevo sol, aproximadamente.

En suma, el presente estudio apoya el enfoque de la adecuación del método PPML dada la consistencia de los resultados (para las dos ecuaciones estimadas), la cual cuenta con el aporte estudios anteriores como los de Silva \& Tenreyro (2006, 2010, 2011); Shepherd \& Wilson (2008). A su vez, los coeficientes estimados por el método PPML son relativamente mejores en términos de signos esperados y la significación estadística.

\section{Conclusiones}

El principal objetivo del estudio fue evaluar los efectos de la integración física, lo que refleja entre otras consecuencias en el aumento en los flujos comerciales y por consiguiente también en la competitividad de los productos ofertados. La idea central es que la integración física promueva un creciente aumento del flujo de comercio entre ambos países, y especialmente en las regiones por donde pasa la carretera Interoceánica.

En particular, las regiones situadas en la zona fronteriza, por donde pasa la carretera por mucho tiempo enfrentan problemas que afectan el comercio, por ejemplo, el caso de la infraestructura deficiente. Estas regiones por estar localizadas lejos de los principales mercados (ya sea Lima en Perú, o San Pablo en el Brasil) enfrentan sistemas logísticos poco desarrollados. En consecuencia, eso afecta los resultados de exportación por medio de los costos, ya que las exportaciones en general, especialmente de alimentos tienden a ser al por mayor. Desde un punto de vista económico, es deseable que recursos sean dirigidos a la mejora de las condiciones de infraestructura (considerados un cuello de botella para el desarrollo agrícola), por lo que el retorno a la sociedad se logrará mediante los beneficios que la misma pueda implicar en el flujo del comercio.

\footnotetext{
${ }^{6}$ Resultados similares para la variable distancia fueron encontrados por Zeynalov (2017) al analizar el comercio de Azerbaiyán con sus principales socios y; Bergstrand et al. (2015) al analizar las fronteras y distancias y su efecto en el flujo del comercio, así como en los acuerdos de integración económica.
} 
Cuadro 6: Resultados de la estimación de la ecuación 1 por varios métodos caso general para todos los estados del Perú que exportan al Brasil

\begin{tabular}{c|cccc}
\hline Variables & Pooled & Efecto Fijo & Efecto Aleatório & PPML \\
\hline \multirow{2}{*}{ LGDP2 } & $0.851^{* * *}$ & $1.618^{* *}$ & $1.359^{* *}$ & $0.092^{* * *}$ \\
& $(0.171)$ & $(0.327)$ & $(0.279)$ & $(0.021)$ \\
\hline \multirow{2}{*}{ Ld2 } & $1.749^{* *}$ & - & $1.124^{*}$ & $0.309^{* *}$ \\
& $(0.998)$ & - & $(2.787)$ & $(0.154)$ \\
\hline \multirow{2}{*}{ Ltc2 } & 0.144 & 0.114 & 0.306 & $0.021^{*}$ \\
& $(1.187)$ & $(0.995)$ & $(0.997)$ & $(0.158)$ \\
\hline \multirow{2}{*}{ Front2 } & $-4.591^{* * *}$ & - & -3.696 & $-0.792^{* *}$ \\
& $(0.547)$ & - & $(1.385)$ & $(0.089)$ \\
\hline Constante & $-18.172^{* *}$ & $-16.736^{* * *}$ & $-21.037^{* * *}$ & $-1.756^{*}$ \\
& $(8.234)$ & $(5.051)$ & $(23.171)$ & $(1.263)$ \\
\hline Observações & 160 & 160 & 160 \\
\hline Grupos Estados & \multicolumn{5}{|c}{10} \\
\hline$R^{2}$ & 0.572 & 10 & 10 \\
\hline Prueba F & $51.75^{* * *}$ & 0.217 \\
\hline Prueba Chow & \multicolumn{5}{|c}{$19.70^{* * *}$} \\
\hline Prueba de Hausman & $12.15^{* * *}$ \\
\hline Prueba LM & \multicolumn{5}{|c}{$8.75^{* * *}$} \\
\hline Log Likelihood & -380.354 & -367.342 \\
\hline Prueba Reset & $9.84^{* * *}$ & $7.52^{* * *}$ \\
\hline
\end{tabular}

Fuente: Elaboración propia.

Nota: Los errores estándar aparecen entre paréntesis. Para los métodos de EF y EA, se informa de la R-cuadrado "within groups"

${ }^{* * *}$ Significancia a $1 \%$; ${ }^{* *}$ Significancia a $5 \%$; ${ }^{*}$ Significancia a $10 \%$.

En general, este estudio contribuye a la teoría del comercio internacional, en concreto el nuevo regionalismo, la misma que se hace relevante en las presentes condiciones, donde la búsqueda por nuevas formas de fortalecer las relaciones bilaterales y el comercio puede servir como impulsores (y servir debate) para el crecimiento de las regiones menos desarrolladas de ambos países. Al mismo tiempo, se evalúa los efectos "distancia", "frontera" y "carretera", siendo eses factores determinantes para la mejora de los flujos comerciales. Además, por medio de este trabajo, se puede asignar un valor tangible a los beneficios de la integración física en el flujo comercial, especialmente para los estados y/o Departamentos por donde pasa la carretera, dada la mejora observada de los flujos comerciales.

Por medio del resultado del "efecto frontera" se evidenció un efecto positivo para Brasil y negativo para Perú. Ante tal, se sostiene que la integración física no mostró ser relevante para las regiones de frontera del Perú, o sea, no existe un beneficio homogéneo en términos de comercio. Es cierto que el comercio en las regiones fronterizas (en el caso del Perú) se ha intensificado, pero no es relevante al comparar con la media de exportación de otros estados. De esa forma, el objetivo de probar que el flujo de comercio en las regiones fronterizas se ha intensificado, fue probado parcialmente.

Considerando el resultado de la variable Ltc2, se determina la importancia del mercado brasileño en términos de atractividad para el mercado peruano, siendo que el contrario no es cierto. En concreto, el comportamiento del tipo 
de cambio siguió el patrón de comportamiento del flujo de comercio, la cual se intensificó en los últimos años.

Finalmente, el resultado empírico a través de la aplicación del modelo econométrico gravitacional reveló que las estimaciones se mostraron adecuadas para evaluar el flujo de comercio en función de las variables dichas en el estudio. Se destaca en este contexto que el método PPML fue el más adecuado para el tipo de muestra utilizado en el estudio (con ceros y valores nulos). Además, considerando la magnitud de los coeficientes obtenidos por el método PPML (los cuales son menores con relación a los métodos alternativos), se argumenta que la no adopción del método PPML puede acarrear en conclusiones erróneas con relación al flujo de comercio, cada vez que se considera el método en cuestión superior a los demás.

En vista de los resultados encontrados, y reforzando lo descrito en parágrafos anteriores (sobre la contribución del estudio), se sugiere para los países que tengan como misión el fortalecimiento bilateral y el fomento de comercio en regiones próximas de frontera y menos desarrolladas, dar mayor consideración a las condiciones de infraestructura y aspectos logísticos, siendo estos determinantes y que al mismo tiempo inciden directamente en el flujo de comercio.

\section{Bibliografía}

Anderson, J. E. (1979), 'A theoretical foundation for the gravity equation', The American Economic Review 69(1), 106-116.

Anderson, J. E. \& Van Wincoop, E. (2003), 'Gravity with gravitas: a solution to the border puzzle', American Economic Review 93(1), 170-192.

Anderson, J. E. \& Van Wincoop, E. (2004), 'Trade costs', Journal of Economic Literature 42(3), 691-751.

Arevalo, J. L. S. \& Merlo, E. M. (2017), 'Análise da competitividade no comércio entre Brasil e Peru: uma abordagem considerando o efeito da integração física', Journal of Globalization, Competitiveness \& Governability/Revista de Globalización, Competitividad y Gobernabilidad/Revista de Globalização, Competitividade e Governabilidade 11(2), 80-97.

Bergstrand, J. H., Larch, M. \& Yotov, Y. V. (2015), 'Economic integration agreements, border effects, and distance elasticities in the gravity equation', European Economic Review 78, 307-327.

Blum, B. S. \& Goldfarb, A. (2006), 'Does the internet defy the law of gravity?', Journal of International Economics 70(2), 384-405.

Breusch, T. S. \& Pagan, A. R. (1980), 'The Lagrange Multiplier test and its applications to model specification in econometrics', The Review of Economic Studies 47(1), 239-253.

Brun, J.-F., Carrère, C., Guillaumont, P. \& De Melo, J. (2005), 'Has distance died? Evidence from a panel gravity model', The World Bank Economic Review 19(1), 99-120. 
Carrere, C. (2006), 'Revisiting the effects of regional trade agreements on trade flows with proper specification of the gravity model', European Economic Review 50(2), 223-247.

Centre d'Études Prospectives et d'Informations Internationales (2016). Metodologia de distância. Disponível em: $<$ http://www.cepii.fr/CEPII/fr/publications/publicat.asp $>$. Acesso em: 14 ago.

Cheng, I.-H., Wall, H. J. et al. (2005), 'Controlling for heterogeneity in gravity models of trade and integration', Federal Reserve Bank of St. Louis Review 87(1), 49-63.

Cipoletta Tomassian, G. (2009), 'Infraestructura fisica e integracion regional'.

de Comercio Exterior y Turismo, M. M. (2016). Base de datos de exportacion. Disponível em: <https://www.mincetur.gob.pe>. Acesso em: 05 ago.

Egger, P. (2005), 'Alternative techniques for estimation of cross-section gravity models', Review of International Economics 13(5), 881-891.

Ethier, W. J. (1998), 'The new regionalism', The Economic Journal 108(449), 1149-1161.

Felipa, P. B. (2012), 'Efecto de la carretera interoceánica en el comércio internacional de Perú y Brasil', Revista Finanzas y Política Económica 4(2), 25-50.

IBGE (2016), 'Banco de dados.', Acesso em 6. Disponible a través de: "<http://www.ibge.gov.br/home/estatistica/economia/contasnacionais/2013/ default.shtm>" "http://www.ibge.gov.br/home/estatistica/economía/contas nacionais/2013/default.shtm>".

INEI (2016). Base de datos. Disponível em: <https://www.inei.gob.pe> Acesso em: 15 Jul. 2016.

Kapuya, T., Chinembiri, E. K. \& Kalaba, M. W. (2014), 'Identifying strategic markets for South Africa's citrus exports', Agrekon 53(1), 124-158.

Linnemann, H. (1966), 'An econometric study of world trade flows', Amsterdam, North Holland .

Magee, C. S. (2008), 'New measures of trade creation and trade diversion', Journal of International Economics 75(2), 349-362.

MDIC (2016). Base de datos. Disponível em: <http://www.mdic.gov.br>. Acesso em: 15 Jul. 2016.

Mier, M. Á. D. (2008), 'Las Distorsiones al comercio de productos agrícolas causadas por las exportaciones subvencionadas: el caso de la Unión Europea, los Estados Unidos de América y sus repercusiones en México y Brasil'.

Oliveira, A. C. (2014), 'Do velho ao novo regionalismo: evolução das políticas conjuntas para o desenvolvimento planejado da América Latina'. 
Otor, S. A. (2017), 'Japan's official development assistance and exports to Asian countries: the donor's perspective', Institutions and Economies pp. 60 91.

Porto, P. C. S., Canuto, O. \& Morini, C. (2015), The impacts of trade facilitation measures on international trade flows, The World Bank.

Ramsey, J. B. (1969), 'Tests for specification errors in classical linear leastsquares regression analysis', Journal of the Royal Statistical Society, Series B (Methodological) pp. 350-371.

Reis, M., Azevedo, A. F. Z. \& Lélis, M. T. C. (2014), 'Os efeitos do novo regionalismo sobre o comércio', Estudos Econômicos (São Paulo) 44(2), 351-381.

Shepherd, B. \& Wilson, J. S. (2008), Trade facilitation in ASEAN member countries: measuring progress and assessing priorities, The World Bank.

Sierralta, A. (2014), 'Teoría evolutiva del comércio internacional', Universidad ESAN, Lima-Peru. .

SIICEX (2016). Base de datos de exportacion. Disponível em: <http://www.siicex.gob.pe>. Acesso em: 30 Jul. 2016.

Silva, J. S. \& Tenreyro, S. (2006), 'The log of gravity', The Review of Economics and Statistics 88(4), 641-658.

Silva, J. S. \& Tenreyro, S. (2010), 'On the existence of the maximum likelihood estimates in Poisson regression', Economics Letters 107(2), 310-312.

Silva, J. S. \& Tenreyro, S. (2011), 'Further simulation evidence on the performance of the Poisson pseudo-maximum likelihood estimator', Economics Letters 112(2), 220-222.

SUNAT (2016). Base de datos de exportacion. Disponível em: <http://www.sunat.gob.pe>. Acesso em: 30 Jul. 2016.

Tinbergen, J. \& Hekscher, A. (1962), Shaping the world economy. Suggestions for an international economic policy., Twentieth Century Fund.

United Nations (2016). Base de dados de exportação. Disponível em: <http://comtrade.un.org/data/>. Acesso em: 12 ago.

Weeks, D. V. (2014), Determinantes do investimento estrangeiro direto bilateral: uma abordagem do ambiente de negócios, $\mathrm{PhD}$ thesis, Universidade de São Paulo.

Zeynalov, A. (2017), 'The gravity of institutions in a resource-rich country: the case of Azerbaijan', International Economics and Economic Policy 14(2), 239-261. 
